\title{
Hearing and Life Quality Assessment in Post-Language Patients Following Cochlear Implant
}

\author{
Giselle de Martin Truzzi, Raquel Andrade Lauria, Alexandre Caixeta Guimarães, \\ Sílvia Badur Curi, Arthur Menino Castilho, Jorge Rizzato Paschoal, Luciane Calonga, \\ Walter Adriano Bianchini, Guilherme Machado de Carvalho* \\ Otology, Audiology and Implantable Ear Prostheses Ear, Nose, Throat and Head \& Neck Surgery Department, \\ Campinas University, São Paulo, Brazil \\ Email: otorrino@fcm.unicamp.br, ${ }^{*}$ gisellemar@ig.com.br
}

Received 16 November 2015; accepted 1 December 2015; published 4 December 2015

Copyright (C) 2015 by authors and OALib.

This work is licensed under the Creative Commons Attribution International License (CC BY). http://creativecommons.org/licenses/by/4.0/

(c) (i) Open Access

\begin{abstract}
Introduction: Hearing loss affects millions of people worldwide, leading some of those to economic impairment, social segregation, and a worse quality of life. Cochlear implants have shown advantages over other treatment alternatives, for it provides considerable hearing gains and therefore greater social interaction possibilities to the affected individuals. Objectives: Speech perception testing and the World Health Organization's Quality of Life Questionnaire (WHOQOL) are used to assess the hearing outcomes and the quality of life of post-lingual patients with severe/profound bilateral neurosensorial hearing loss treated with cochlear implant (Medel). Methods: Speech perception tests are applied to selected subjects prior to and following the cochlear implant using Med-EL CI; the questionnaires assessing quality of life are answered afterwards. Results: There is an improvement in the audiometric thresholds, and the patients present a good quality of life after cochlear implant. Conclusion: The evaluation of these subjects permits to conclude that there are improvements in hearing, and that patients perceive their quality of life to be good after being subjected to cochlear implant.
\end{abstract}

\section{Keywords}

Cochlear Implants, Satisfaction, Quality of Life, Hearing Loss

Subject Areas: Otorhinolaryngology

\footnotetext{
${ }^{*}$ Corresponding author.
}

How to cite this paper: de Martin Truzzi, G., Lauria, R.A., Guimarães, A.C., Curi, S.B., Castilho, A.M., Paschoal, J.R., Calonga, L., Bianchini, W.A. and de Carvalho, G.M. (2015) Hearing and Life Quality Assessment in Post-Language Patients Following Cochlear Implant. Open Access Library Journal, 2: e2178. http://dx.doi.org/10.4236/oalib.1102178 


\section{Introduction}

Hearing loss affects about three hundred and sixty million people worldwide (5.3 percent of the world population), nine percent of those being children and $91 \%$ adults [1].

Hearing loss may lead those individuals to isolation and exclusion from social interaction, depression, cognitive impairment and bring a negative economic impact to society, with higher levels of unemployment in this population. Within the population with disabling hearing impairment, there are individuals with severe/profound bilateral sensorineural hearing loss, which can be subjected to some therapies, such as learning signal language and using personal sound amplification devices, like hearing aids (HA) [1] [2].

However, HA are very often unable to bring significant hearing benefits to those patients and even after learning sign language, the individuals maintain great social isolation. Thus, there can be advantages in using cochlear implants (CI) as treatment for those hearing losses, as they permit achieving greater hearing, social and economic benefits [3]. Around 324,200 people worldwide have received CI, and it has aided those individuals with hearing impairments [4].

This article aims to describe achievements in sound perception of post-lingual patients and to evaluate their quality of live by WHOQOL.

\section{Subjects and Methods}

Retrospective clinical study with analysis of medical records of patients who underwent surgical treatment for severe/profound hearing loss with model cochlear implants were performed.

The medical literature review was performed using the Mesh Terms "hearing loss; cochlear implants; quality of life" at Pubmed and Scopus database.

\subsection{Patient Selection}

The sample was composed by patients who underwent cochlear implant Medel, followed in hearing care service university hospital in the last five years.

Only patients who underwent audiological and electrophysiological testing with the same staff and equipment and also had complete data in the medical records were included in the sample.

\subsection{Inclusion Criteria}

Inclusion criteria were: sensorineural hearing loss (severe/profound), normal otoscopy, absence of middle ear disease, absence of acoustic reflex, absence in ABR waves and imaging (MRI/CT; Philips ${ }^{\circledR}$, Netherlands) showing the presence of the cochlear nerve and excluding retrocochlear disturbances.

Hearing aids were used in all subjects before treatment and when no benefits were showed it was indicated the cochlear implants (have sensorineural bilateral hearing loss with little or no benefit from HA (less than 40\% of auditory discrimination in monosyllables), have pure-tone thresholds $\geq 80 \mathrm{~dB}$ hearing loss, have had stable hearing loss for at least the past two years, and lastly pass a psychological examination ensuring they had realistic expectations about the potential benefits of receiving a cochlear implant. All subjects underwent pure tone audiometry (PTA) and speech tests, pre- and postoperatively.

All patients who did not complete these criteria were excluded from the study.

\subsection{Audiological Evaluation}

Audiological tests were performed including impedanciometry, speech and pure tone audiometry. The tests were performed using an audiometer AC30-SD25 (Interacoustics ${ }^{\circledR}$, Denmark), calibrated according to ISO 389 standards/64.

For ABR, which were repeated at least two times, we used the device AT-235 (Interacoustics ${ }^{\circledR}$, Denmark).

The hearing impairment was stratified in mild, moderate, severe or profound hearing loss, according to Goodman [5].

\subsection{Speech Perception Tests}

Preoperatively, all subjects took a speech perception test at the same day of their implantation. We used a speech 
perception sentence test based on Bevilacqua et al. [6]. Subjects did the test with their hearing aids on, in a quiet place.

Postoperatively, all subjects repeated the speech perception test at least one year after CI. Tests were done in subject's best-aided condition: CI-only. The same audiologist conducted all the pre and postoperative tests.

\subsection{Quality of Life Evaluation}

The patients also completed the World Health Organization's Quality of Life Questionnaire (WHOQOL), in its validated version to Brazilian Portuguese. Data obtained from the tests and questionnaires were then analysed and are presented next [7] [8].

The World Health Organization Quality of Life (WHOQOL) project was initiated in 1991. The aim was to develop an international cross-culturally comparable quality of life assessment instrument. It assesses the individual's perceptions in the context of their culture and value systems, and their personal goals, standards and concerns. The WHOQOL instruments were developed collaboratively in a number of centres worldwide, and have been widely field-tested [7] [8].

The WHOQOL-BREF instrument comprises 26 items, which measure the following broad domains: physical health, psychological health, social relationships, and environment. The WHOQOL-BREF is a shorter version of the original instrument that may be more convenient for use in large research studies or clinical trials [7] [8].

\subsection{Statistical Analysis}

The data were analyzed using descriptive analysis, with production of means, medians, standard deviation tabs.

T-student test was used to compare the pre-operative and postoperative speech perception scores. The confidence Interval was of $95 \%$, and $p$-value $<0.05$ was considered significant.

\subsection{Ethical Considerations}

This study was previously approved by the Research Ethics Committee of the Faculty of Medical Sciences of the University of Campinas and all subjects signed the consent informed term.

\section{Results}

A total 22 patients met the study's inclusion criteria, with ages, ranging between 21 and 73 . Seven of those patients presented abrupt bilateral hearing loss, while fifteen had a progressive bilateral hearing loss. Table 1 shows this detail's data.

Among the analysed patients, 21 reportedly had family support to undergo the procedure, and during the whole pre-and post-surgical procedure as well. Psychologists prior to the implant evaluated all subjects, and none revealed any data that could exclude them from receiving the implants. No patient presented complications during or following the surgical intervention.

The following Table 2 presents the data of the speech perception tests performed before and following the cochlear implant. Out of the analysed patients, all patients' achievement in speech perception after the procedure, and half of the patients had an improvement equal to or greater than $86 \%$.

\begin{tabular}{ccc}
\hline Table 1. Clinical data of the studied patients. & \multicolumn{2}{c|}{ Details } \\
\hline & Patients/values & $41 \%$ \\
Male & 9 & $59 \%$ \\
Female & 13 & $31.8 \%$ \\
Bilateral HL-abrupt & 7 & $68.2 \%$ \\
Bilateral HL-progressive & 15 & min $21-$ max 73 \\
Age (years) & 34.5 & $0 \%$ \\
Surgical complications & 0 & min 0 - max 56\% median $0 \%$ SD 0.15 \\
Speech test pre op (HA+) & $8 \%$ & $\min 24$ - max 100\% median 85\% SD 0.25 \\
Speech test post op (CI+)
\end{tabular}

Ps.: HA = hearing aids; $\mathrm{CI}$ = cochlear implants; HL = hearing loss; SD: standard deviation. 
Table 2. Speech Perception Test (SPT) before and after the Cochlear Implant (CI) procedure.

\begin{tabular}{|c|c|c|}
\hline Subjects & SPT pre op & SPT post op \\
\hline 1 & $0 \%$ & $96 \%$ \\
\hline 2 & $0 \%$ & $44 \%$ \\
\hline 3 & $4 \%$ & $46 \%$ \\
\hline 4 & $0 \%$ & $24 \%$ \\
\hline 5 & $0 \%$ & $82 \%$ \\
\hline 6 & $8 \%$ & $96 \%$ \\
\hline 7 & $0 \%$ & $32 \%$ \\
\hline 8 & $40 \%$ & $90 \%$ \\
\hline 9 & $0 \%$ & $100 \%$ \\
\hline 10 & $0 \%$ & $40 \%$ \\
\hline 11 & $0 \%$ & $96 \%$ \\
\hline 12 & $56 \%$ & $100 \%$ \\
\hline 13 & $0 \%$ & $98 \%$ \\
\hline 14 & $0 \%$ & $96 \%$ \\
\hline 15 & $0 \%$ & $96 \%$ \\
\hline 16 & $0 \%$ & $54 \%$ \\
\hline 17 & $0 \%$ & $44 \%$ \\
\hline 18 & $0 \%$ & $82 \%$ \\
\hline 19 & $12 \%$ & $92 \%$ \\
\hline 20 & $0 \%$ & $88 \%$ \\
\hline 21 & $46 \%$ & $74 \%$ \\
\hline 22 & $28 \%$ & $90 \%$ \\
\hline
\end{tabular}

The average of the speech test (with HA) at the pre op time was $8 \%$ (min 0: max 56\%; median $0 \%$; standard deviation 0.15 ) and in the post op time (with CI) was $75 \%$ (min $24 \%$ : max 100\%; median $85 \%$, standard deviation 0.25). This data had a statistical significance $(p<0.05)$.

When asked if they believed that implanting the device was the best available option for treatment, $90 \%$ answered they believed strongly or very strongly that it was the best treatment option they had for their hearing loss.

When asked if they thought their hearing aids compensated their handicap, there were reports (shown in Figure 1) of 16 patients stating it compensated their handicap "very much" or "an extreme amount".

Data obtained from the completion of the WHOQOL questionnaire were analysed after the interviews. Some of the results on the patients' quality of life are presented in Figures 2-4.

\section{Discussion}

To perform the present study, data from patients treated by a single surgical team using a single brand of CI were analysed in order to reduce biases potentially inflicted by the use of different devices or surgical techniques. However, the small number of subjects can be a limitation of this study.

In general, most patients believe CI was the best available option to treat their hearing loss. Analysing the questionnaire, one can perceive the majority of patients were satisfied with their health, with their quality of life and felt their lives were meaningful. 
Do you think your hearing aids compensate your handicap?

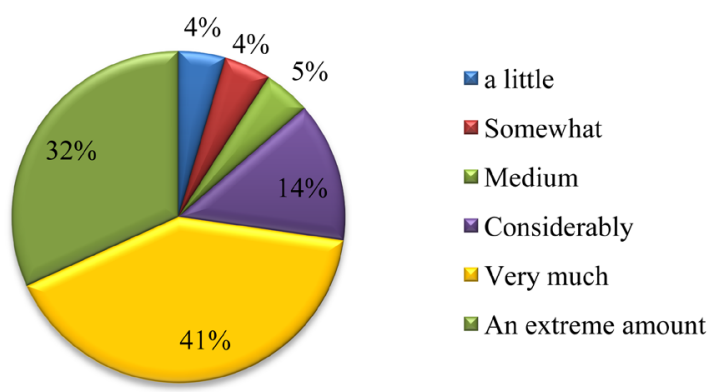

Figure 1. Do you think your hearing aids compensate your handicap?

\section{How would you rate your quality of life?}

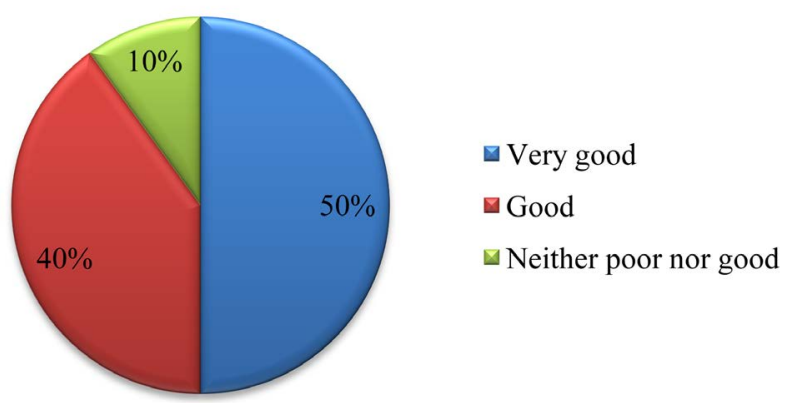

Figure 2. How would you rate your quality of life?

How satisfied are you with your health?

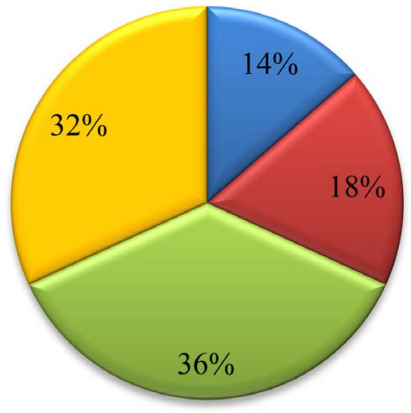

$\square$ Dissatisfied

$\square$ neither satisfied nor dissatisfied

$\square$ Satisfied

$\square$ Very Satisfied

Figure 3. How satisfied are you with your health?

To what extent do you feel your life to be meaningful?

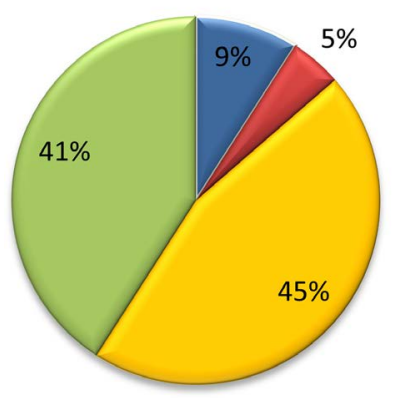

$\square$ A little

A moderate amount

$\square$ Very much

$\square$ An extreme amount

Figure 4. To what extent do you feel your life to be meaningful? 
Even though the answers for this questionnaire haven't been obtained before the surgery in order to allow comparisons with the current responses, it is inferred that the cochlear implant had a positive impact in the subjects' lives, considering hearing loss usually brings negative impacts to the affected patients.

Although there is no standard questionnaire on quality of life to assess it in patients subjected to CI, research show it is improved by the surgical procedure [9] [10]. However, there are may be differences between the individual's quality of life scores and the audiological responses, given that they are not necessarily correlated [11].

A proper psychological evaluation and prepare prior to the surgical act may be determinant to a positive perception of the patients' own quality of life [12].

Analysing the patients, one can observe there was a gain at speech perception in most of the patients following cochlear implant. When assessing the quality of life, it is seen most individuals reported having a good quality of life and considered the cochlear implant to be a beneficial procedure to correct their hearing loss.

\section{Conclusion}

In this studied population, the $\mathrm{CI}$ is related to a great improvement at speech perception and with positive answers at WHOQOL questionnaire.

\section{References}

[1] Deafness and Hearing loss. World Health Organization. www.who.int/mediacentre/factsheets/fs300/en

[2] Budenz, C.L., Cosetti, M.K., Coelho, D.H., Birenbaum, B., Babb, J., Waltzman, S.B. and Roehm, P.C. (2011) The Effects of Cochlear Implantation on Speech Perception in Older Adults. Journal of the American Geriatrics Society, 59, 446-453. http://dx.doi.org/10.1111/j.1532-5415.2010.03310.x

[3] Penaranda, A., et al. (2012) Beneficios económicos del implante coclear para la hipoacusia sensorineural profunda. Revista Panamericana de Salud Pública, 31, 325-331.

[4] Cochlear Implants. National Institute of Deafness and Other Communication Disorder. http://www.nidcd.nih.gov/health/hearing/pages/coch.aspx

[5] Goodman, A. (1965) Reference Zero Levels for Pure-Tone Audiometers. ASHA, 7, 262-273.

[6] Bevilacqua, M.C., Banhara, M.R., da Costa, E.A., Vignoly, A.B. and Alvarenga, K.F. (2008) Brazilian Portuguese Hearing in Noise Test. International Journal of Audiology, 47, 364-365. http://dx.doi.org/10.1080/14992020701870205

[7] (2002) Whoqol-Hiv Instrument, Mental Health: Evidence and Research Department of Mental Health and Substance Dependence. World Health Organization, Geneva. http://apps.who.int/iris/bitstream/10665/77774/1/WHO_MSD_MER_Rev.2012.01_eng.pdf?ua=1

[8] (2002) Quality of Life-BREF (WHOQOL-BREF). World Health Organization, Geneva. http://www.who.int/substance abuse/research tools/whoqolbref/en/

[9] Gaylor, J.M., Raman, G., Chung, M., Lee, J., Rao, M., Lau, J. and Poe, D.S. (2013) Cochlear Implantation in Adults: A Systematic Review and Meta-Analysis. JAMA Otolaryngology—Head \& Neck Surgery, 139, 265-272. http://dx.doi.org/10.1001/jamaoto.2013.1744

[10] Castilho, A.M., Pauna, H.F., Fernandes, F.L., Bonhin, R.G., Guimarães, A.C., de Melo, T.M., Cheng, M., Sartorato, E.L., de Carvalho, G.M. and Paschoal, J.R. (2015) HiFocus Helix ${ }^{\mathrm{TM}}$ Electrode Insertion: Surgical Approach. BMC Research Notes, 15, 304.

[11] Capretta, N.R. and Moberly, A.C. (2015) Does Quality of Life Depend on Speech Recognition Performance for Adult Cochlear Implant useRs?Laryngoscope, in press. http://dx.doi.org/10.1002/lary.25525

[12] Kobosko, J., Jedrzejczak, W.W., Pilka, E., Pankowska, A., Skarzynski, H. (2015) Satisfaction with Cochlear Implants in Postlingually Deaf Adults and Its Nonaudiological Predictors:Psychological Distress, Coping Strategies, and SelfEsteem. Ear and Hearing, 36, 605-618. http://dx.doi.org/10.1097/AUD.0000000000000179 